\title{
Study the Relationship between Pavement Surface Distress and Roughness Data
}

\author{
Muhammad Mubaraki \\ Department of Civil Engineering, Jazan University, Saudi Arabia
}

\begin{abstract}
In this paper, pavement sections from the highway connected Jeddah to Jazan were selected and analyzed to investigate the relationship between International Roughness Index (IRI) and pavement damage including; cracking, rutting, and raveling. The Ministry of Transport (MOT) of Saudi Arabia has been collecting pavement condition data using the Road Surface Tester (RST) vehicle. The MOT measures Roughness, Rutting (RUT), Cracking (CRA), raveling (RAV). Roughness measurements are calculated in terms of the International Roughness Index (IRI). The IRI is calculated over equally spaced intervals along the road profile. Roughness measurements are performed at speed between at 80 kilometers per hour. Thus RST vehicle has been used to evaluate highways across the country. The paper shows three relationships including; cracking (CRA) verses roughness (IRI), rutting (RUT) verses IRI, and raveling (RAV) verses IRI. Also, the paper developed two models namely; model relates IRI to the three distress under study, and model relates IRI to ride quality. The results of the analysis claim at $95 \%$ confidence that a significant relationship exist between IRI and cracking, and raveling. It's also shown that rutting did not show significant relationship to IRI values. That's leads to conclude that the distresses types: cracking and raveling may possibly be described as ride quality distresses at different level of significant. Rutting distress described as non-ride quality type's distresses.
\end{abstract}

\section{Introduction}

Pavement evaluation is a process by which field surveys and testing are carried out to characterize the condition of an existing pavement structure, both structurally and functionally. The structural condition of a pavement refers to its ability to support the current and future traffic loadings, whereas the functional condition refers to its ability to provide a safe, smooth, and quiet riding surface for the traveling public (Hass et al 1994).

Network-level evaluations are conducted on pavement sections within the network of pavements for which the agency is responsible, with the general purpose to document current conditions, to identify projects for maintenance, preservation and rehabilitation, to help prioritize projects and allocate budgets, and to help determine funding needs. In addition, the collection of performance data on a pavement network over time provides a valuable tool for tracking pavement performance as well as a mechanism for developing performance models that can be used to predict future conditions (both with and without the application of treatments).

Visual condition surveys are generally included determining type of distress, its severity, its extent and location. There are

Many methods for conducting pavement condition surveys, as there are agencies involved. Pavement condition index (PCI) ranges from zero to 100 , where 100 represents excellent pavement condition. The PCI values are calculated based on pavement distress type, severity and quantity collected through visual inspection. To collect the appropriate distress data an inspector is sent to a particular pavement section to record the existing distress types, severities and quantities. The information is collected by actually walking through the section. The procedure provides detailed information on pavement section condition. However, considering the size of the city pavement network the procedure is very time consuming and very costly.

Roughness is primary a measure of riding quality of the road pavement surface. Pavement roughness is very much related to pavement serviceability, which is a measure of physical characteristics of pavement surface. Roughness measurement systems that currently used can be grouped into profilometric, vehicle response and subjective evaluation. Profilometric methods are the most accurate and best suited for detailed analysis.

Roughness measurements are calculated in terms of the International Roughness Index (IRI). The IRI is calculated over equally spaced intervals along the road profile, the IRI computation method converts the longitudinal and vertical profile data into a vehicle motion response using mathematical model. The IRI value is expressed as the units of displacement over units of length. Roughness measurements are performed at speed between 
(40 to 50) kilometers per hour. Thus, roughness measurements collected in relatively very short time without excessive cost (Mubaraki, 2014).

\section{Research significant}

Application of pavement evaluation of pavements should be on a well-planned basis and be an integral part of the overall pavement management system. Pavement roughness data fulfills a wide variety of needs in the management function. These needs occur at network and project levels, for rural and urban road pavements. For example, at the network level, roughness data provides a functional evaluation of the pavement surface which can be used in project selection and programming. The roughness data evaluation helps in construction quality control and evaluation of rehabilitation options (Hass et al 1994).

Application of pavement distress data has recognized by pavement engineers as an important parameter for quantifying the quality of pavement surface. It is important at both the network and projects levels of pavement management system. For example, at both levels, the pavement distress information is useful in selecting appropriate treatments. At the network level, the concern is with determining what class of treatment is required for example, continued routine maintenance or resurfacing. At the project level, the concern is with estimating the extent and the methods of pavement repair such as to patch a certain area of alligator cracking (Hass et al 1994).

Roughness is primary a measure of riding quality of the road pavement surface. Pavement roughness is very much related to pavement serviceability, which is a measure of physical characteristics of pavement surface. The applicability of pavement roughness as a predictor variable of pavement condition index is a vital in pavement evaluation and maintenance to set criteria or trigger levels for different types of maintenance. On other hand, the interaction between pavement roughness and pavement distress types is very important to distinguish between ride quality distress type and non-ride quality type.

Therefore, the expected achievement of the research will greatly assist in determining maintenance needs by defining the types of distress that most probability will be accrued, determining the effective timing of applying the maintenance needs by defining the time after which the rate of deterioration will increase drastically, and help the municipalities in setting priority index for the budget and the maintenance program, and hopefully the results can help in local research in the areas of pavement maintenance.

\section{Objectives}

The main objective of this study is to study the relationship between pavement condition evaluation and roughness measurements and examine the effect of pavement distress types on pavement roughness for the Jazan entire road network.

\section{Methodology}

\subsection{Correlation of IRI and type of distress}

The Pearson correlation analysis was conducted to estimate the correlation factor and correlation hypothesis and to find out whether a significant statistical relation between IRI and types of distress exist. The statistical hypothesis test displays a p- value to determine the significant effect of a distress on IRI values (Park et al, 2004).

\subsection{IRI and distress model}

The modeling procedure used in the analysis is based on statistical regression. The basic form of regression equation is as follows:

$y=b 0+b 1 X$; Where:

Y: Dependent Variable (response)

$\mathrm{X}$ : Independent Variable

b0, b1: regression coefficients

To attain the analysis procedure, a spreadsheet is used to examine and study the data. The spread sheet program was used to tabulate the data, draw the charts (graphs) that describe the trend of the collected data, building the models and find out the correlations and the relationship between pavement roughness (IRI) and types of distress.

The IRI and distress model is constructed in order to predict types of distress in terms of a distress behavior as a function of pavement roughness (IRI). The procedure involved using sufficient regression techniques to construct models linking IRI and a distress values. The most suitable model was then selected based on proper statistical tests. The process of constructing the regression model was based on three types of regression functions (Myers and Keying, 2011):

1-Linear regression approach, assuming the relation between the two variables is linear. The regression equation is in the form Distress $=b 0+b 1 * I R I$. In this equation the regression coefficients are $b 0$ and $b 1$.

2-Logarithm nonlinear relation approach, assuming the relation between IRI and a distress is nonlinear. The distress was considered as a function of logarithm of IRI. The nonlinear relation is then transformed to linear regression by calculating new parameter called Log (IRI). The regression equation is in the form: Distress = $b 0+b 1 \log$ (IRI). In this equation the regression coefficients are b0 and b1.

3-Quadratic nonlinear relation approach, assuming the relation between IRI and a distress is nonlinear. The distress was considered as a function of quadratic of IRI.

4-Distress $=\mathrm{f}$ IRIa. The nonlinear relation is transformed to linear regression by calculating new parameter called IRIa; where: a is constant. The regression equation is in the form: Distress $=b 0+b 1$ IRIa. In this equation the regression coefficients are b0 and $\mathrm{b} 1$.

After the regression equations are constructed, a process of testing was used. The aim of the testing process is to find out the best suitable model that represents the 
given data. The analysis procedure was based on three main statistical regression tests, which are:

1-Test of hypothesis (T-test).

2-Coefficient of multiple determination (R2)

3-Analyze of Variance Test (ANOVA - Test)

The T-test of hypothesis is incorporated to study the relationship between each of regression coefficients (b0, b1) and the response variable at certain degree of confidence (assumed 95\% in our study).Hence the test was conducted for all of the regression coefficients in the regression model. The coefficient of multiple determinations (R2) was used to illustrate the adequacy of a fitted regression model. The $\mathrm{R} 2 \mathrm{is}$ a percentage indicates the proportion of the response variable variations explained by the regression model. The coefficient takes values from 0 (no correlation exists between variables) to 100 (perfect correlation between variables). The Analysis Of Variance test (ANOVA) is powerful tool used to investigate the relationship between the response variable (a distress in this study) and the independent variables (IRI). The ANOVA test extends the t-test for more general null hypothesis to estimate the quality of the regression line. That is whether the variation in distress readings is dependent on the IRI readings. This proves the significant evidence to accept or reject the regression model. (Myers and Keying, 2011).

\subsection{Site selection}

The study involves the analysis of roughness and distress data from a highway connects Jeddah city with Jazan city. Many readings from the surveys have been deployed to this study.

\subsection{Data collection}

For the selected highway, cracking, rutting, raveling, and road surface profiles were obtained from Ministry of Transport (MOT), Pavement Management System (PMS) database. Pavement distress values and roughness values were available for 2009, 2011, and 2013. The pavement distress and roughness data was available for $400 \mathrm{~km}$ for each $\mathrm{km}$ intervals. Detailed distress data in the form of distress type, distress density, and as well as measurements of roughness.

\section{Data analysis}

\subsection{Cracking vs.IRI}

Fig. 1 presents graph relation between IRI and cracking density. It could be noticed that the IRI never exceeded above 5 in most data points, and that is due to the fact that data collecting van drivers are instructed to avoid driving heavily deformed segments of the road. Also, for a cracking distress between $4 \% \& 6 \%$, the IRI values were clustered around " 1 ". This irregularity most probably represents segments of the road with a very good IRI but had longitudinal cracks between the road and its paved shoulders. The graph verifies the positive relation between the cracks and IRI values. However, the graph does not show a clear cut decision about the linearity of the relation. The correlation coefficient of IRI and patching density is 0.500 and the $P$ value is equal to.019. The positive sign of the coefficient indicates a positive relation between cracking density and IRI. The value of correlation factor indicates that about $50 \%$ of the IRI and cracks density observations were distinguished by the linear relation. The best representation for the cracking data was by logarithmic formula and it's as Cracking $=8.6+2.7 \log I R I$.

In the hypothesis test, the $P$-value $=0.019$, therefore there is sufficient evidence at $95 \%$ confidence level of the relationship between IRI and patching density. The result of the statistical test doesn't comply with previews experiences that point toward cracks distress is on ride quality type of distress at all severity levels.

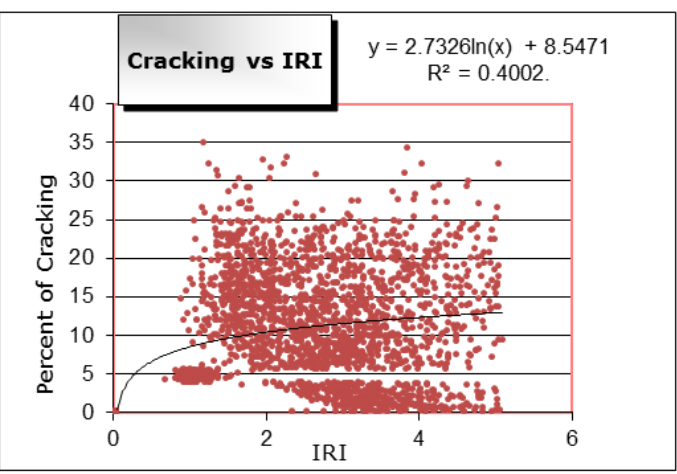

Figure 1. IRI and cracking distress density relationship.

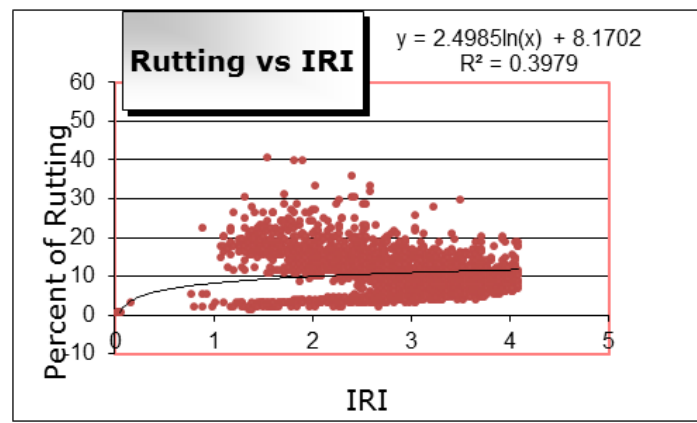

Figure 2. IRI and rutting density relationship.

\subsection{Rutting vs. IRI}

The relationship between IRI and rutting density is presented in Fig. 2. The Graph Indicates A Positive relation between the rutting and IRI values. There is no clear cut decision about the linearity of the relation. The Correlation coefficient of IRI and rutting density is 0.464 and P-value is 0.151 .

The value of the correlation coefficient equals 0.464 indicates that only about $46.4 \%$ of the IRI and running density observation were not able by the linear relation. For this reason, logarithm nonlinear relation approach was used. The regression model was as; Rutting $=8.2+2.5$ $\log$ IRI. The P-value is 0.15 which is higher than the 0.05 level of significant; therefore there is no sufficient evidence of the relationship between IRI and rutting density. 


\subsection{Ravelling vs. IRI}

Fig. 3 represents a graph relation between IRI and raveling distress density. The raveling data appears in 50 pavement sections. The graph indicates a positive relation between raveling and IRI. The value of correlation factor indicates that about $45 \%$ of the IRI and raveling density observation were distinguished by the linear relation. The best model formula is as Raveling $=28.2+9.00 \log$ IRI.

In the hypothesis test the P-value equals 0.006 . Therefore there is sufficient evidence at $95 \%$ confidence level of the relationship between IRI and raveling density.

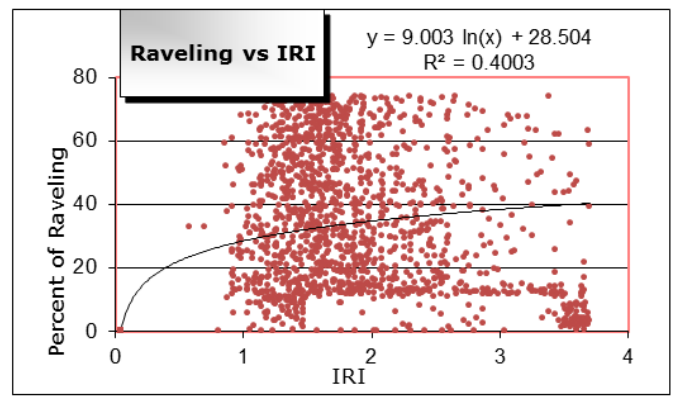

Figure 3. IRI and raveling density relationship.

\section{Discussion}

The effect of all the distress types that appears in the study area on the IRI reading was investigated. The study indicated the riding quality related types of distress. The relationship between IRI and distress types has been investigated through woke statistical tests:

- The individual Correlation hypothesis test between IRI and each distress types.

- The regression coefficients test for overall regression model relating IRI and distresses types.

The-correlation-hypothesis- test for cracking, rutting and raveling, demonstrates that $\mathrm{P}$ is zero except rutting. Therefore cracking and raveling and I have significantrelationship. Hence the result of the analysis climate 95\% confidence that a significant relationship exist between IRI and cracking and raveling. Furthermore, the low correlation factors for all the three types of distress showed that there is no clear cut decision about the linearity of the relation. Therefore, the best representation is a nonlinear equation. In this study, it was found the logarithm shows reasonable fitting for the data.

Those results were proved through the statistical tests on the overall model between IRI and types of distress. The regression coefficients test compute $p$ values for each regression coefficient. The regression coefficients for cracking and raveling are equal zero. This indicates a significant linear relationship between IRI and each regression coefficients. Obviously, different distress types related to IRI differently. It was found that from the values of the correlation factors, that patching and depression have the highest correlation to IRI. While the raveling has less correlation, cracking has the least relation to IRI. It was also found that the correlation factors for all the distressed against IRI were not exceed $50 \%$, which mean that less than 50 percent of these relationships are definable by the relationship.
This relationship is not strong enough for pavement condition to be used as measure for IRI. Hence, the result of both correlation and regression coefficients tests indicates at $95 \%$ confidence that cracking, and raveling are rid equality type distressed. However, it's not realistic to conclude that IRI may completely reflect pavement distress conditions. The rutting, pointed toward no significant relationship to IRI values. The $\mathrm{P}$ Values (which larger than 0.05level of significance) of rutting demonstrate that this types of distress has no strong evidence to significant relationship with IRI.

Those results were proved through the statistical tests on the overall model between IRI and type of distress. The regression coefficients test computed $p$-values for each regression coefficient. The regression coefficient for rutting is greater than 0.05 level of significance, indicating no significant linear relationship between IRI and rutting. Hence, the result of both correlation and regression coefficients tests indicates at 95\% confidence that rutting is not ride quality type of distress, and that IRI could not reflect rutting. There as on for these results may be the fact that running is in the longitudinal profile of pavement not detected roughness machine.

\section{Conclusion}

Pavement sections from the street network of Jazan city have been selected to evaluate correlation of three types of distress including cracking, rutting, and raveling distress densities values to IRI values. Based on the results, correlations, and statistical models that relate IRI values to the distress density values were established.

A statistical correlation analysis performed to analyze the interaction between IRI in pavement distresses. The aim of this analysis was to determine the riding quality related type's distresses. The correlation statistical test was us to determine the correlation between distress density and IRI values.

The model related IRI and distresses types were estimated in the pavement sections. The relationship between IRI and distresses types has been investigated through correlation hypothesis test and regression coefficients test for overall model relating IRI and distresses types.

The results of the analysis claim at $95 \%$ confidence that a significant relationship exist between IRI and cracking, and raveling. It's also shown that rutting did not show significant relationship to IRI values. That's leads to conclude that the distresses types: cracking, and raveling may possibly be describe $\mathrm{d}$ as ride quality distresses at different level of significant. Rutting distress described as non-ride quality type's distresses.

\section{References}

1. R. Hass, W. Hudson, and J. Zaniewnski, Modern Pavement Management, Krieger publishing, (1994)

2. M. Mubaraki, Identification of Pavement Distress Types and Pavement Condition Evaluation Based on Network Level Inspection for Jazan City Road 
Network, The Journal of Engineering Research (TJER), 11, No. 1, pp 44-54, (2014)

3. M. Mubaraki, A methodology for project-level maintenance for urban road, Journal of TransportICE, 168, 3, pp 239-255, (2015)

4. T. El-Korchi and W. Norman, Visual Appearance of surface Distress in Pavement, TRB No. 1260 Washington, D. C., (2004)

5. L. Aultman and E. Jackson, Model Relating Pavement Quality Measures. Journal of Transportation Research Board, Washington, D. C., (2004)

6. Myers and Keying E. Ye 2011. Probability and Statistics for Engineers and Scientists. (2011)

7. M. Shahin and S. Kohn, Pavement Maintenance Management for Road And Parking Lots, (2002)
8. K. Park, K. Lee, and T. Natacha, Applicability of the International Roughness Index as a Predictor of Asphalt Pavement Condition. Journal of Transportation Research Board, Washington, D. C., (2004)

9. S. Dewan and R. Smith, Estimating IRI from Pavement Distresses to Calculate Vehicle Operating Costs for the Cities and Counties of San Francisco Bay Area. Transportation Research Record 1816, Transportation Research Board; Washington D. C., (2002)

10. J. Lin, J. Yau, and L. Hsiao, Correlation analysis Between International Roughness Index (IRI) and pavement Distress by Neural Network, Journal of Transportation Research Board, Washington, D. C., (2003) 